\section{Síndrome disentérico, insuficiencia cardiaca y anemia grave por tricuriasis. Presentación de un caso y revisión bibliográfica}

Karina Guerrero-Doria, ${ }^{1}$ Mónica Patricia Escobedo-Chávez, ${ }^{2}$ José SevillaBacilio, ${ }^{3}$ David Moncada-Barrón, ${ }^{4}$ Gerardo Flores-Nava ${ }^{5}$

\section{Resumen}

ANTECEDENTES: Trichuris trichiura es un parásito que cuando produce infestación masiva en el humano puede complicarse con anemia severa.

OBJETIVO: reportar el caso de una niña con infestación crónica de tricocéfalos que le produjo una anemia severa (cor anémico).

DESCRIPCIÓN DEL CASO: niña que vive en sitio rural, con alimentación deficiente, geofagia y fecalismo al aire libre. El padecimiento se inició un mes antes de su ingreso con síncopes, astenia y adinamia, disnea progresiva hasta abandonar la escuela y la semana previa al ingreso se agregó diarrea sanguinolenta. En la exploración física: palidez mucocutánea, soplo holosistólico grado V/VI, hepatomegalia, dedos hipocráticos, desnutrición grado II, hemograma con hemoglobina de $2.4 \mathrm{~g} / \mathrm{dL}$. Electrocardiograma normal. Radiografía de tórax con cardiomegalia, índice cardiotorácico 0.57. Coproparasitoscópico positivo a huevos de Trichuris trichiura. Recibió tratamiento con transfusión de concentrado eritrocitario, mebendazol, nitazoxanida y tratamiento nutricional. La evolución fue satisfactoria con incremento ponderal, mejoría hemodinámica y coproparasitoscópico de control negativo.

DISCUSIÓN: el diagnóstico de anemia severa (cor anémico) implica investigar la causa; en este caso, ante el antecedente de que la paciente vive en zona rural y por dos cuadros previos de prolapso rectal y uno de diarrea con sangre se solicitaron coproparasitoscópicos con resultado positivo a tricocéfalos.

PALABRAS CLAVE: anemia, Trichuris trichiura, parasitosis.
${ }^{1}$ Residente de tercer año, Pediatría Médica.

2 Jefa del Departamento de Infectología Pediátrica.

${ }^{3}$ Médico adscrito, Terapia Intensiva Pediátrica.

${ }^{4}$ Adscrito al Laboratorio Clínico.

${ }^{5}$ Jefe de la División de Pediatría Clínica.

Hospital General Dr. Manuel Gea González, Secretaría de Salud, Ciudad de México.

Recibido: 28 de marzo 2016

Aceptado: 28 de abril 2017

Correspondencia

Dr. Gerardo Flores Nava

gerflores50@hotmail.com

Este artículo debe citarse como

Guerrero-Doria K, Escobedo-Chávez MP, Sevilla-Bacilio J, Moncada-Barrón D, Flores-Nava G. Síndrome disentérico, insuficiencia cardiaca y anemia grave por tricuriasis. Presentación de un caso y revisión bibliográfica. Acta Pediatr Mex. 2018;39(2):185-189. 


\section{Dysentery syndrome, heart failure, severe anemia by trichuriasis. Case report and review of the literature.}

Karina Guerrero-Doria, ${ }^{1}$ Mónica Patricia Escobedo-Chávez, ${ }^{2}$ José SevillaBacilio, ${ }^{3}$ David Moncada-Barrón, ${ }^{4}$ Gerardo Flores-Nava ${ }^{5}$

\begin{abstract}
BACKGROUND: Trichuris trichiura is a parasite when massive infestation in humans occurs may be complicated by severe anemia.

OBJECTIVE: To report the case of a child with chronic infestation of Trichuris trichiura that produced severe anemia and heart failure.

CASE DESCRIPTION: School girl living in rural areas, with poor nutrition, geophagia and outdoor defecation. She began prior to joining with syncope, fatigue and weakness, progressive dyspnea to leave school and one week prior month bloody diarrhea was added. Physical examination: mucocutaneous pallor, blow holosystolic grade V/VI, hepatomegaly, hippocratic fingers, malnutrition Grade II, hemoglobin $2.4 \mathrm{~g} / \mathrm{dl}$, electrocardiogram with normal result, chest radiography with cardiomegaly, cardiothoracic ratio 0.57 . Coproparasitoscopic positive to Trichuris trichiura eggs. She was treated with concentrated erythrocyte transfusion, mebendazole, nitazoxanide and nutritional management. The evolution was satisfactory with weight gain, and hemodynamic improvement coproparasitoscopic negative control.
\end{abstract}

DISCUSSION: The diagnosis of severe anemia and heart failure involves investigating the cause, in this case against the background that the patient lives in rural areas and twice rectal prolapse and one of bloody diarrhea was requested coproparasitoscopic Trichuris trichiura positive result.

KEYWORDS: anemia; Trichuris trichiura; parasitic diseases
Correspondence

Dr. Gerardo Flores Nava

gerflores50@hotmail.com

\section{INTRODUCCIÓN}

Cerca de 800 millones de personas están parasitadas por Trichuris trichiura alrededor del mundo; de éstos, 100 millones corresponden a Latinoamérica y el Caribe. Se encuentra entre las enfermedades despreciadas Neglected Tropical Diseases (NTDs) y afecta con mayor frecuencia a los niños de 1 a 4 años de edad (86.2\%) seguidos por los escolares de 5 a 10 años (56.3\%). En la mayoría de los casos esta infección es asintomática, pero las infecciones masivas se manifiestan con diarrea crónica, desnutrición y anemia. ${ }^{1}$ Este nematodo tiene distribución geográfica amplia en México, especialmente en zonas tropicales rurales y húmedas, es favorecido por el inadecuado manejo de excretas y desechos, geofagia y mala higiene de manos. ${ }^{2}$ 


\section{DESCRIPCIÓN DEL CASO}

Niña indígena de 9 años de edad proveniente del municipio de Huautla de Jiménez en Oaxaca, vivienda rural, alimentación deficiente basada en tortillas y frijol, geofagia positiva y fecalismo al aire libre. Con antecedente de diarrea sanguinolenta, tenesmo, pujo rectal y prolapso rectal a los 7 años de edad; tratada en el sistema de salud de su localidad (centro de salud) con reducción manual del prolapso y fármacos no especificados. El prolapso y su reducción manual se repitieron dos meses antes de su ingreso; un mes después tuvo varios síncopes que duraban aproximadamente treinta segundos, acompañados de mareo, astenia, adinamia y disnea progresiva hasta pequeños esfuerzos con imposibilidad para realizar labores cotidianas, lo que la obligó a abandonar la escuela donde cursaba el cuarto grado.

Una semana antes de su ingreso se sumó diarrea sanguinolenta de predominio nocturno, asociada con pujo y tenesmo. Acudió a urgencias del hospital. La paciente pesó $14 \mathrm{~kg}$ (percentil <5\% para su edad) que corresponde a un déficit de $56 \%$ para la edad, talla de $100 \mathrm{~cm}$ (percentil $<5 \%$ para la edad) con déficit de $27 \%$, por lo que se consideró con desnutrición crónica agudizada en la clasificación de Waterlow. ${ }^{3}$ Taquicárdica y polipneica, oximetría de pulso $87 \%$. Exploración física: marcada palidez mucocutánea generalizada, en tórax soplo holosistólico plurifocal grado $\mathrm{V} / \mathrm{VI}$, en abdomen distensión, hepatomegalia $5 \times 5 \times 5 \mathrm{~cm}$ abajo del reborde costal, sin circulación colateral, extremidades con escaso panículo adiposo, hipocratismo en dedos de manos y pies, acrocianosis, Ilenado capilar 6 segundos. Biometría hemática: anemia severa hipocrómica, microcítica, hiponatremia, hipopotasemia, hipoalbuminemia; hemoglobina $2.40 \mathrm{~g} / \mathrm{dL}$, hematócrito $9.20 \%$, anisocitosis +++ , microcitosis +++, hipocromía +++, hierro $27 \mu \mathrm{g} /$ $\mathrm{dL}$, transferrina $466 \mathrm{mg} / \mathrm{dL}$, ferritina $7.20 \mathrm{ng} /$ $\mathrm{mL}$, sodio $129 \mathrm{mEq} / \mathrm{L}$, potasio $2.4 \mathrm{mEq} / \mathrm{L}$, cloro
$89 \mathrm{mg} / \mathrm{dL}$, calcio $8.2 \mathrm{mg} / \mathrm{dL}$, magnesio $1.6 \mathrm{mg} /$ $\mathrm{dL}$, proteínas totales $6.14 \mathrm{~g} / \mathrm{dL}$, albúmina $2.25 \mathrm{~g} /$ dL, globulina $3.89 \mathrm{~g} / \mathrm{dL}$, relación albúmina/ globulina 0.58 , sangre oculta en heces + , coprocultivo negativo y coproparasitoscópicos (3) con abundantes huevos de Trichuris trichiura (Figura 1).

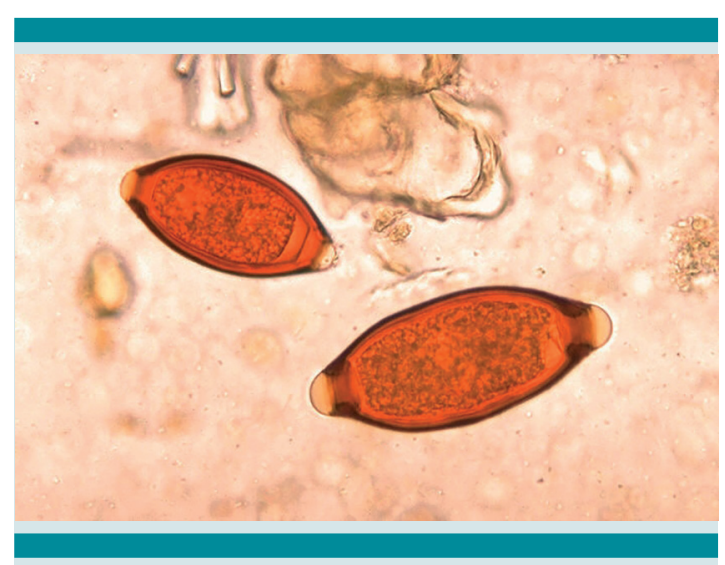

Figura 1. Fotografía microscópica de uno de los coproparasitoscópicos de la paciente, se aprecian dos huevecillos típicos de Trichuris trichiura.

Se realizó un electrocardiograma con resultado normal. La radiografía de tórax mostró cardiomegalia, índice cardiotorácico de 0.57 , cefalización del flujo y congestión parahiliar bilateral.

Durante su hospitalización fue evaluada por cardiología pediátrica donde se descartó cardiopatía y se emitió el diagnóstico de insuficiencia cardiaca por anemia severa (cor anémico), se transfundió concentrado eritrocitario a dosis de $10 \mathrm{~mL} / \mathrm{kg}$ de peso, en dos ocasiones, con siete días de diferencia y con vigilancia estrecha de su estado hemodinámico.

Se administraron dos antiparasitarios: mebendazol (100 mg) y nitazoxanida (15 mg/kg/día) durante 3 días y sulfato ferroso en dosis de $2 \mathrm{mg} /$ kg/día. Al aliviarse de las complicaciones por desnutrición se le dio una dieta adecuada para la fase aguda de la desnutrición. Permaneció 
hospitalizada durante 21 días, su evolución fue con mejoría hemodinámica e incremento ponderal progresivo; la hemoglobina subió a $12.50 \mathrm{~g} / \mathrm{dL}$. Al alta pesó 17.5 kilogramos y sus coproparasitoscópicos de control fueron negativos. Se envió a su domicilio con dieta específica de recuperación nutricional y desparasitación cada seis meses, incluido todo su núcleo familiar.

\section{DISCUSIÓN}

La paciente de este caso integró el diagnóstico de síndrome disentérico por Trichuris trichiura, común en áreas endémicas de algunos países y bien documentado en la bibliografía médica. ${ }^{4}$ Quizá fue correctamente diagnosticada pero mal tratada en su lugar de origen, ya que al parecer no se llevó a cabo un tratamiento completo o un seguimiento a largo plazo, por lo que se trasladó hasta la Ciudad de México, con sus propios medios, a un hospital de segundo nivel de atención médica donde se recibió con insuficiencia cardiaca que ponía en peligro su vida y anemia muy grave (concentración de hemoglobina sumamente baja, de hasta $2 \mathrm{~g} / \mathrm{dL}$ ) que fue tratada en forma estrecha con dopamina, dobutamina y transfusiones de paquete globular con buena respuesta clínica. Esa cifra de hemoglobina no se encontró reportada en otros pacientes similares, pero un estudio publicado en el año 2008, en niños con anemia severa (hemoglobina menor de $5 \mathrm{~g} / \mathrm{dL}$ ) menciona media y desviación estándar de $3.6 \pm 0.8 \mathrm{~g} / \mathrm{dL}$; sin embargo, no comentan cuál fue el valor más bajo de hemoglobina. Dicho estudio se llevó a cabo en un país africano donde la anemia severa es una de las principales causas de hospitalización y con mayor contribución a la muerte. La parasitosis coexiste en estos pacientes, pero principalmente por malaria. ${ }^{5}$ No es un caso único a escala mundial pero en México son pocos los reportes similares. ${ }^{1}$

Trichuris trichiura es un parásito de amplia distribución en las regiones tropicales y húmedas. La lombriz adulta mide $4 \mathrm{~cm}$, tiene forma de látigo con un segmento caudal grueso que se encuentra libre en la luz intestinal y un segmento anterior que se fija en la mucosa mediante un estilete que penetra la mucosa hasta los capilares. La hembra produce hasta 20,000 huevos diarios que requieren ser embrionados en la tierra antes de ser infectantes, por eso no se trasmite de persona a persona; se ubica en el colon y ciego pero en infestaciones puede abarcar a todo el intestino grueso.

El cuadro clínico se caracteriza por episodios de colitis disentéricas y, en ocasiones, por prolapso rectal por hipotonía de los músculos perineales y relajación del esfínter anal. Se puede acompañar de anemia microcítica e hipocrómica como consecuencia de la implantación del parásito en la mucosa con un sangrado continuo y pérdida de hasta $0.005 \mathrm{~mL}$ de sangre por lombriz por día y no por hematofagia como en otros nematodos. ${ }^{6}$ El diagnóstico se confirma en el laboratorio por método coproparasitoscópico cuantitativo seriado visualizando los quistes característicos del parásito. A largo plazo produce deterioro nutricional, detención del crecimiento y dificultad en el aprendizaje; son pocos los casos en la bibliografía reportados con anemia grave, concentraciones de hemoglobina menores a $5 \mathrm{~g} / 100 \mathrm{~mL}$ e insuficiencia cardiaca que afecte las actividades de la vida cotidiana del paciente.? La mayor tasa de infección se encuentra entre la población de 1 a 4 años (86.2\%) seguida por la población en edad escolar (a la cual pertenece la paciente) que es de $58.1 \%$; todo esto es favorecido por el bajo nivel socioeconómico, la ausencia de agua potable, viviendas con piso de tierra, inadecuado manejo de excretas y desechos, geofagia y mala higiene de manos, características coexistentes en el caso expuesto.

La paciente tenía síntomas de parasitosis intestinal desde dos años antes de su ingreso, manifestada por episodios de disentería que en dos oportunidades se complicó con prolapso rectal; es decir, un síndrome disentérico por Trichuris 
trichiura $^{4,8}$ sin recibir tratamiento adecuado. Tuvo un sangrado del tubo digestivo bajo lento y progresivo, frecuente en este tipo de infección. Un reporte menciona que a mayor número de huevecillos del parásito, en el coproparasitoscópico, mayor grado de anemia en los pacientes. ${ }^{9}$ En nuestra paciente no se pudo determinar el número de huevecillos por cuestiones técnicas.

El hipocratismo en manos y pies es un dato clínico que traduce mala oxigenación tisular distal y se encuentra con frecuencia en este síndrome, aunque no es patognomónico. ${ }^{10}$ También hubo detención del crecimiento: a pesar de tener 9 años de edad su estatura y peso estaban por debajo de la centila 5 y más de dos desviaciones estándar de la media, las medidas correspondían a las de una niña de 4 años. Otro aspecto muy importante es que la paciente dejó de asistir a la escuela el mes previo a su ingreso por los síntomas de la anemia grave. Estas complicaciones pudieron haberse evitado con un tratamiento adecuado de la parasitosis inicialmente, lo que está al alcance de cualquier médico en el primer nivel de atención médica. La paciente egresó a los 21 días con incremento de peso y desaparición de los síntomas cardiovasculares; se ha reintegrado a su comunidad. Los tratamientos adecuados para la insuficiencia cardiaca, la anemia y la parasitosis resultaron en un beneficio para la paciente.

\section{REFERENCIAS}

1. Carrada BT. Trichuriosis: Epidemiología, diagnóstico y tratamiento. Rev Mex Pediatr. 2004;71:299-305.

2. Quihui-Cota L, Morales-Figueroa GG, Esparza-Romero J, Valencia ME, Astiazarán-García H, Méndez RO y col. Trichuriasis and low-iron status in schoolchildren from Northwest Mexico. Eur J Clin Nutr. 2010;64:1108-1115.

3. Marquez GH, García SVM, Caltenco SML, García VEA, Marquez FH, Villa RAR. Clasificación y evaluación de la desnutrición del paciente pediátrico. El Residente. 2012:7;659-696.

4. Khuroo MS, Khuroo MS, Khuroo NS. Trichuris dysentery syndrome: a common cause of chronic irondeficiency anemia in adults in an endemic area. Gastrointestinal endoscopy. 2010;71:200-204.

5. Calis CJJ, Piri KS, Faragher EB, Brabin BJ, Bates I, Cuevas LE. Severe anemia in malawin children. $N$ Engl J Med. 2008;358:888-899.

6. Layrisse M, Aparcedo L, Martinez-Torres C; Roche M. Blood loss due to infection with Trichuris trichiura. Ann Trop Med Parasit. 1967;16:613-619.

7. Bernal RR. Parasitología. En García AJA, Valencia MPF, editores. Urgencias en Pediatría Hospital Infantil de México. 6a ed, México, McGraw Hill Interamericana Editores S.A de C.V. 2011, p. 1340-1346.

8. Bernard NH, Barrientos MAR, Ramos MHJ, Ismael CG. Prolapso rectal en Honduras. Reporte de caso y revisión de la literatura. Rev Med Hondur. 2014;82:115-118.

9. HF Yang, CC Wang, CF Hu, CC Hsieh, HS Lee,SJ Chen, HC Fan. Importance of Considering Trichuris trichiura Infection in Infant Presenting with Acute and Substantial Bloody Diarrhea: A Case Report and Literature Review. J Med Sciences. 2012;32:309-312.

10. Bowie MD, Morison A, Ireland JD, Duys PJ. Clubbing and whip worm infestation. Arch Dis child. 1978;53:411-413. 